\title{
Effect of Surface Stresses on Microwave Surface Resistance and Its Impact for Cavity Perturbation Measurements
}

\author{
Nicholas Clark, Greg Shaw, and Adrian Porch
}

\begin{abstract}
For microwave characterization of materials over a wide temperature range, it is often desirable to perform cavity perturbation measurements at elevated temperatures. Careful cavity design, involving the minimization of electrical contacts, ensures high measurement stability as the temperature varies. However, examining the Q-factor of a cylindrical cavity resonator during heating has shown that heat treatment can permanently modify the surface resistance of an aluminum surface. X-Ray diffraction measurements of Al-6082 confirm that the source of change can be due to changes in surface stress and the appearance of solution precipitates. Here we discuss the necessity to pretreat microwave cavities to stabilize their Q-factors in order to reliably measure material properties via cavity perturbation across a wide temperature range.
\end{abstract}

Index Terms-Cavity resonators, microwave measurement, perturbation methods, stress measurement.

\section{INTRODUCTION}

D URING the heat treatment of a metal, a number of effects can act to modify its apparent conductivity. For instance, impurities precipitating out of solution or crystalline modifications such as a reduction in dislocation density.

A changing conductivity caused by annealing can have a significant impact on a number of microwave spectroscopy techniques. Whilst the effects discussed and measured here can be applied to these different techniques, here we concentrate on the implications for cavity resonators and the perturbation technique in particular. To the authors' knowledge, these effects have not been widely discussed or measured before.

It is often desirable to conduct perturbation experiments across a range of sample temperatures. Instead of implementing a complex sample heating system, it is often convenient to place the entire measurement fixture within an oven. However, this presents a challenge since the resonant frequency and quality factor $(\mathrm{Q})$ used to measure the sample properties are themselves modified by temperature.

One of the key advantages often touted by the cavity perturbation technique is its relatively low cost and simplicity.

Manuscript received May 17, 2017; accepted June 28, 2017. Date of publication September 25, 2017; date of current version October 5, 2017. This work was supported by the U.K. Engineering and Physical Sciences Research Council through the ICASE program in partnership with Renishaw Plc. (Corresponding author: Nicholas Clark.)

N. Clark and A. Porch are with the Centre for High Frequency Engineering, Cardiff University, Cardiff CF24 3AA, U.K. (e-mail: clarkns@cardiff.ac.uk; porcha@cardiff.ac.uk).

G. Shaw is with the Cardiff Catalysis Institute, School of Chemistry, Cardiff University, Cardiff CF10 3AT, U.K. (e-mail: shawg3@ cardiff.ac.uk).

Color versions of one or more of the figures in this paper are available online at http://ieeexplore.iee.org.

Digital Object Identifier 10.1109/LMWC.2017.2745486
Cavities can easily be constructed in two relatively simple pieces but, since cost is a key motivator, a tradeoff between performance and cost is usually made. This often means the use of a widely available and easily machined metal alloys such as 6082 Aluminum.

Slow heating of an aluminum alloy, as has been done here, will cause diffuse solute impurities to precipitate from the solid solution. Whilst these precipitates can initially act to increase electron scattering and reduce conductivity, generally, as heating progresses, it is expected that the nucleation sites will grow in size, becoming less numerous, and act to increase conductivity [1]-[3]. It is noted that the potential effects are complex and strongly dependent on the alloy constituents and any pretreatment of the material.

An additional effect is the gradual removal of residual stresses introduced during machining. The machining responsible is usually termed as cold working, as it occurs below the recrystallization temperature [4]. Residual stresses are known to reduce a metal's conductivity due to the increased scattering by lattice dislocations [5]-[7]. Annealing the metal will reduce residual stresses by removal of these lattice distortions and dislocations [8]. This effect will be most pronounced for annealing temperatures above the recrystallization temperature; this is typically under $300{ }^{\circ} \mathrm{C}$ for the most commonly used alloys of aluminum. The use of eddy current techniques, usually at relatively low frequencies, is commonplace to ascertain the effects of applied and residual stresses [9], [10]. These will be compounded at microwave frequencies due to the skin effect, since the stresses induced by machining will be present in the cut surfaces.

\section{BACKGROUND}

We are interested in extending the technique of microwave cavity perturbation over a wide range of temperatures where the upper limit is typically set by the availability of suitable coaxial cables at around $250{ }^{\circ} \mathrm{C}$.

When a metal is heated, it will physically expand and its resistivity will rise. These changes have the effect of reducing resonant frequency and reducing resonant $\mathrm{Q}$.

In order to compensate for this change, the cavity can undergo an empty calibration. This can be quite accurate if the calibration temperature profile matches the measurement temperature profile but in other circumstances requires accurate measurement of the cavity temperature which may significantly reduce the sensitivity of the measurement. An alternative is to indirectly monitor the temperature via a 




Fig. 1. (a) $\mathrm{TM}_{010}$ current distribution. (b) Circumference split configuration. (c) Diameter (clamshell) split configuration. (d) Constructed 6082-Al cylindrical cavity with "clamshell" construction-internal radius $4.6 \mathrm{~cm}$ and internal height $4 \mathrm{~cm}$. $\mathrm{TM}_{010}$ at $2.5 \mathrm{GHz}$.

reference mode. A suitable reference mode will feature an electric and magnetic field node at the sample space so that it is unaffected by the presence of a sample. Since any changes induced by temperature can be attributed directly to the cavity itself, using measurements of the reference mode, the unperturbed state of the measurement mode can be calculated.

An additional consideration is the location of any mechanical joint in the cavity. Traditional manufacturing techniques necessitate that cavity resonators are built in two or more pieces. If current flows across this joint, it forms an electrical contact and will contribute to (i.e., reduce) the resonator Q. When the cavity is heated and thermal expansion occurs, stresses can be put on the joint, thus modifying its electrical properties. Experience, detailed here, has shown that this effect can be very nonlinear. Careful design can, however, practically eliminate this problem for certain resonator configurations.

A popular configuration is the cylindrical cavity resonator operating in the $\mathrm{TM}_{010}$ mode for dielectric measurement where end surface currents flow radially. Therefore, a split placed along its diameter is preferable to the one placed around the circumference as shown in Fig. 1.

Whilst this split configuration is advantageous for the measurement mode, it does not solve the problem for the obvious reference mode in this configuration, $\mathrm{TM}_{210}$, as revealed later.

The main alloying elements of the alloy used here, 6082-T6, are Magnesium, Silicon, and Manganese. The T6 designation indicates that the metal has been solution treated to ensure that alloying elements are fully dissolved into the aluminum matrix before artificial aging at $100{ }^{\circ} \mathrm{C}-200{ }^{\circ} \mathrm{C}$. The precipitation behavior of 6XXX series Aluminum alloys has been widely studied and is relatively complex but is expected to end in $\mathrm{Mg}_{2} \mathrm{Si}$ zones [11].

Treatment at a relatively low temperature $\left(<200{ }^{\circ} \mathrm{C}\right)$ is high enough to cause significant stress recovery but without significant chemical change, since any initial clustering will have occurred during the T6 treatment. Higher temperatures should cause significant and measureable precipitation of $\mathrm{Mg}_{2} \mathrm{Si}$ as reported in [12].

\section{Cavity Measurements}

A cavity was constructed and is shown in Fig. 1(d). Resonant frequency and $\mathrm{Q}$ were measured in transmission using the lorentzian fit method [13]. Capacitive coupling

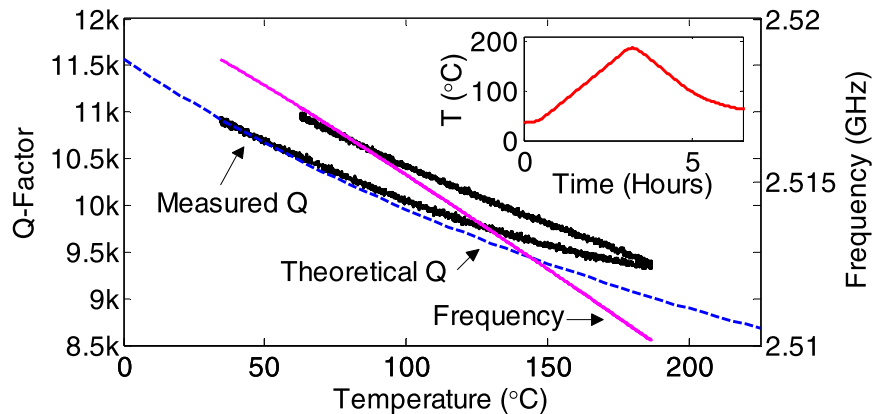

Fig. 2. Frequency and Q-factor of $\mathrm{TM}_{010}$ mode during heat treatment of empty 6082-Al cavity.

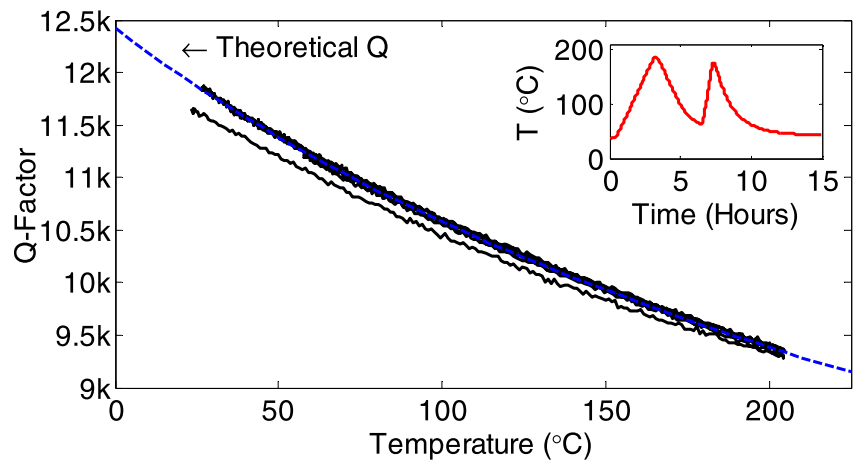

Fig. 3. Q-factor of $\mathrm{TM}_{010}$ mode during repeated heat treatment of 6082-Al cavity.

is provided by two open circuit coaxial probes on the top cavity surface. Coupling is kept weak to ensure minimal Q loading and practically eliminate any potential nonlinearity from changing coupling. In this case, the insertion loss was around $50 \mathrm{~dB}$, corresponding to a loaded and unloaded $Q$ difference of $0.3 \%$. Fig. 2 shows that the resonator parameters measured during a heating cycle with the entire cavity placed within a regular oven (Memmert UF30Plus). Temperature was measured by a PT100 RTD in contact with the cavity surface. A theoretical $Q$ is calculated using a previously derived expression for $Q$ [14] and considering the thermal coefficient of resistivity of aluminum. Since the theoretical prediction will overestimate $Q$, due to surface roughness effects and sample and coupling holes, a fixed offset is used to match the measured and theoretical values at $25^{\circ} \mathrm{C}$.

Fig. 2. shows the frequency change due to thermal expansion is predictable and repeatable. In contrast, the Q-factor shows some nonlinear behavior, diverging from the theoretical prediction and returning to a higher value; once heated, repeating the ramp shows a reduced effect and, with a third repetition, no appreciable further change is observed (Fig. 3). Also, by reducing the fixed offset, the theoretical prediction now closely matches the measured results.

The implication of this result is clear: in order to conduct perturbation measurements across a wide range of temperature, pretreatment of the cavity is required so to stabilize the $Q$.

In addition, measuring the Q-factor of the reference mode $\left(\mathrm{TM}_{210}\right)$ reveals some interesting behavior: strongly nonlinear behavior at low temperatures in the initial ramps. 


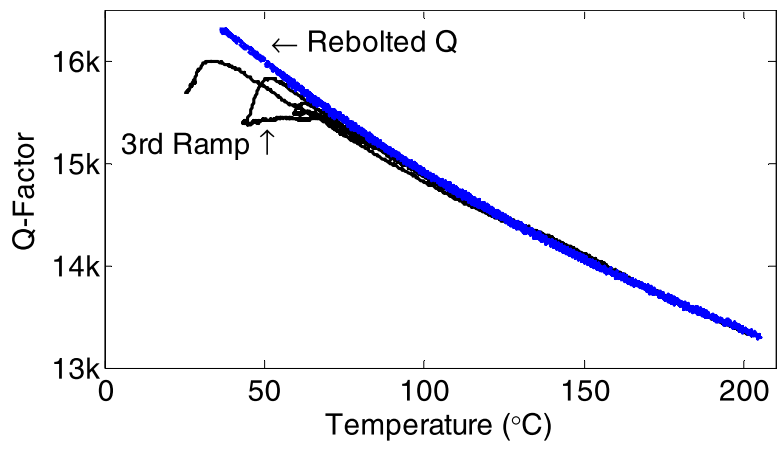

Fig. 4. Q-factor instability of $\mathrm{TM}_{210}$ during third heat treatment cycle and stability after subsequent reassembly.

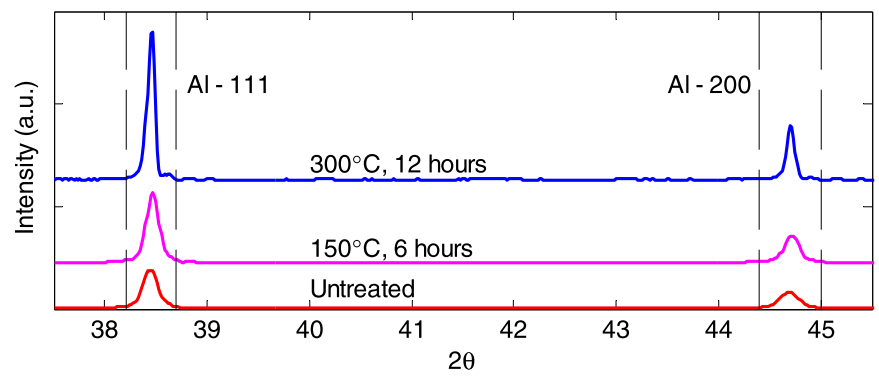

Fig. 5. Heat treated Al-6082 XRD spectra showing primary Al peaks growing and narrowing with heat treatment.

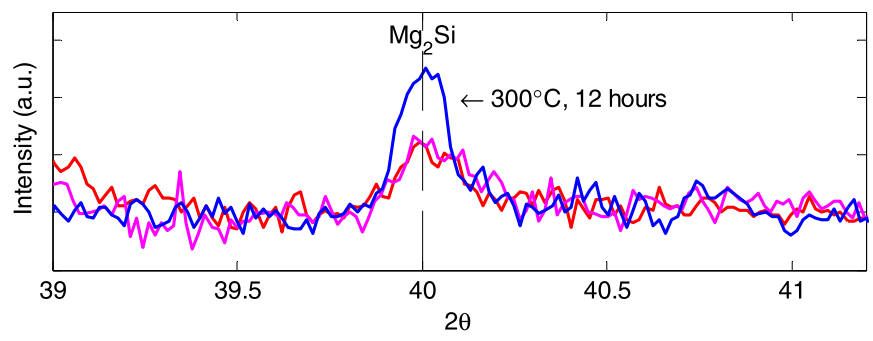

Fig. 6. Heat treated Al-6082 XRD spectra showing primary $\mathrm{Mg}_{2} \mathrm{Si}$ peak. Significant change is only seen in the $300{ }^{\circ} \mathrm{C}$ case.

However, disassembling and rebolting the cavity solved the instability as shown in Fig. 4. This demonstrates the difficulty which can be encountered for cavity designs where current is forced to flow across bolted joints.

It is also noted that typical measurements permit frequency changes, pessimistically, to the order of $100 \mathrm{~Hz}$ to be measured. In this case, a change of $100 \mathrm{~Hz}$ would be caused by an expansion of $1.85 \times 10^{-9} \mathrm{~m}$ which would be the result of a $1.76 \mathrm{mK}$ change in temperature.

\section{X-RAY DIFFRACTION}

In order to understand the encountered behavior and verify the hypothesis of reducing lattice stress and solute precipitation, three cylindrical samples $(\varnothing=3 \mathrm{~mm}, h=1 \mathrm{~mm})$ were prepared in Al-6082 suitable for X-Ray diffraction. After heat treatment, the subsequent XRD spectra reveal the two competing effects which act to modify the conductive losses of the metal.
First, as indicated by the increasing peak intensity and narrowing peak width, as shown in Fig. 5, increasing crystallinity as the heat treatment reduces crystalline defects and surface stresses created during machining. It is clear that the treatment at $150{ }^{\circ} \mathrm{C}$ is not enough to completely stress relieve the metal. Second, the appearance of $\mathrm{Mg}_{2} \mathrm{Si}$ precipitate is shown in Fig. 6. As expected, this does not appear when treated only at $150{ }^{\circ} \mathrm{C}$.

\section{CONCLUSION}

Cavity perturbation has proven to be a flexible materials characterization technique. With a simple oven, dielectric or magnetic characterization can be undertaken across a wide temperature change. However, care must be taken to ensure the $Q$ is stable across the desired temperature range. Whilst the cause of instability can be attributed to stress relief and solute precipitation, it is difficult to decouple these competing effects, although, in Al-6082, the latter can be avoided by keeping the maximum temperature below $150{ }^{\circ} \mathrm{C}$.

Furthermore, utilizing a reference mode, very small temperature changes can be measured, typically with much more sensitivity than traditional temperature probes.

\section{REFERENCES}

[1] M. A. Salazar-Guapuriche et al., "Correlation of strength with hardness and electrical conductivity for aluminium alloy 7010," Mater. Sci. Forum, vols. 519-521, pp. 853-858, Jul. 2006.

[2] Y. Liu, D. M. Jiang, and W. J. Li, "The effect of multistage ageing on microstructure and mechanical properties of 7050 alloy," J. Alloys Compounds, vol. 671, pp. 408-418, Jun. 2016.

[3] G. Lin, Z. Zhang, H. Wang, K. Zhou, and Y. Wei, "Enhanced strength and electrical conductivity of $\mathrm{Al}-\mathrm{Mg}-\mathrm{Si}$ alloy by thermo-mechanical treatment," Mater. Sci. Eng. A, vol. 650, pp. 210-217, Jan. 2016.

[4] S. Adeosun, O. Sekunowo, S. Balogun, and L. Osoba, "Effect of deformation on the mechanical and electrical properties of aluminummagnesium alloy," J. Minerals Mater. Characterization Eng., vol. 10, no. 6, pp. 553-560, 2011.

[5] G. R. Metcalfe, "The use of electrical conductivity measurements in detecting heat and fire damage in aircraft structure," in Proc. IEE Colloq. NDT Technol. Aerosp., Jan. 1990, pp. 2-1-2-4.

[6] M. Sinvani, A. J. Greenfield, A. Bergmann, M. Kaveh, and N. Wiser, "Effect of annealing on the temperature dependence of the electrical resistivity of aluminium," J. Phys. F, Met. Phys., vol. 11, no. 1, p. 149, 1981.

[7] C. S. Çetinarslan, "Effect of cold plastic deformation on electrical conductivity of various materials," Mater. Des., vol. 30, no. 3, pp. 671-673, 2009.

[8] O. P. Agnihotri, "Dislocation densities in heavily cold-worked copper and aluminium," Brit. J. Appl. Phys., vol. 17, no. 5, pp. 603-605, 2002.

[9] M. Zergoug, G. Kamel, and N. Boucherou, "Mechanical stress analysis by eddy current method," J. Amer. Sci., vol. 4, no. 4, pp. 4-9, 2008.

[10] F. C. Schoenig, Jr., A. J. Soules, H. Chang, and J. J. DiCillo, "Eddy current measurements of residual stresses induced by shot peening in titanium Ti6Al4V," Mater. Eval., vol. 55, pp. 22-26, Jan. 1995.

[11] A. K. Gupta, D. J. Lloyd, and S. A. Court, "Precipitation hardening processes in an $\mathrm{Al}-0.4 \% \mathrm{Mg}-1.3 \% \mathrm{Si}-0.25 \% \mathrm{Fe}$ aluminum alloy," Mater. Sci. Eng. A, vol. 301, no. 2, pp. 140-146, 2001.

[12] N. Kumar, P. N. Rao, R. Jayaganthan, and H.-G. Brokmeier, "Effect of cryorolling and annealing on recovery, recrystallisation, grain growth and their influence on mechanical and corrosion behaviour of $6082 \mathrm{Al}$ alloy," Mater. Chem. Phys., vol. 165, pp. 177-187, Sep. 2015.

[13] P. J. Petersan and S. M. Anlage, "Measurement of resonant frequency and quality factor of microwave resonators: Comparison of methods," J. Appl. Phys., vol. 84, no. 6, p. 3392, 1998.

[14] R. E. Collin, Foundations for Microwave Engineering. New York, NY, USA: McGraw-Hill, 1966. 